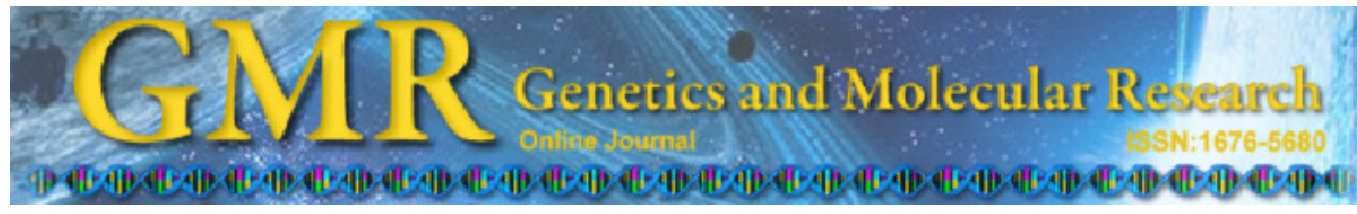

Short Communication

\title{
Plasticity of Corynebacterium diphtheriae pathogenicity islands revealed by PCR
}

\author{
S.C. Soares ${ }^{1}$, F.A. Dorella ${ }^{1}$, L.G.C. Pacheco ${ }^{2}$, R. Hirata Jr. ${ }^{3}$, \\ A.L. Mattos-Guaraldi ${ }^{3}$, V. Azevedo ${ }^{1}$ and A. Miyoshi ${ }^{1}$ \\ ${ }^{1}$ Departamento de Biologia Geral, Instituto de Ciências Biológicas, \\ Universidade Federal de Minas Gerais, Belo Horizonte, MG, Brasil \\ ${ }^{2}$ Departamento de Biointeração, Universidade Federal da Bahia, Salvador, BA, Brasil \\ ${ }^{3}$ Faculdade de Ciências Médicas, Universidade do Estado do Rio de Janeiro, \\ Rio de Janeiro, RJ, Brasil \\ Corresponding author: A. Miyoshi \\ E-mail: miyoshi@icb.ufmg.br
}

Genet. Mol. Res. 10 (2): 1290-1294 (2011)

Received December 14, 2010

Accepted April 25, 2011

Published June 28, 2011

DOI 10.4238/vol10-2gmr1211

\begin{abstract}
Despite the existence of a vaccine against diphtheria, this disease remains endemic and is reemerging in several regions due to many factors, including variations in genes coding for virulence factors. One common feature of virulence factors is their high concentration in pathogenicity islands (PAIs), very unstable regions acquired via horizontal gene transfer, which has lead to the emergence of various bacterial pathogens. The 13 putative PAIs in Corynebacterium diphtheriae NCTC 13129 and the reemergence of this disease point to the great variability in the PAIs of this species, which may reflect on bacterial life style and physiological versatility. We investigated the relationships between the large number of PAIs in $C$. diphtheriae and the possible implications of their plasticity in virulence. The GenoFrag software was used to design primers to analyze the genome plasticity of two pathogenicity islands of the reference strain (PiCds 3 and 8) in 11 different strains. We found that $\mathrm{PiCd} 3$ was absent in only two
\end{abstract}


strains, showing genes playing putative important roles in virulence and that only one strain harbored $\mathrm{PiCd} 8$, due to its location in a putative "hotspot" for horizontal gene transfer events.

Key words: Corynebacterium diphtheriae; Pathogenicity islands; Genome plasticity; PCR; GenoFrag; Horizontal gene transfer

Since the development of a vaccine based on the inactivation of the diphtheria toxin (DT), coded by the tox gene, the cases of diphtheria over the world have decreased drastically (Popovic et al., 2000). However, despite the existence of immunization with DT vaccine, the disease remains endemic in several regions (Mattos-Guaraldi et al., 2003), and more than 150,000 new cases of diphtheria were reported in the former Soviet Union in the 1990s (Nakao et al., 1996; Popovic et al., 2000; Sharma et al., 2007).

Although the reasons for the reemergence of diphtheria in those countries are not clearly elucidated, there are hypotheses that consider several possibilities, including changes in biochemical properties due to potential variations in genes coding for virulence factors harbored by the pathogen Corynebacterium diphtheriae, such as tox and DT repressor $(d t x R)$ (Nakao et al., 1996; Popovic et al., 2000; Mattos-Guaraldi et al., 2003). According to Nakao et al. (1996), variations in the tox gene could lead to inadequate immune response from vaccination with the toxoids originated from the vaccinal strain, $C$. diphtheriae $P W 8$, in people who had contact with the new variant strains.

The toxoid-based nature of the vaccine along with the fact that the tox gene occurs in a pathogenicity island (PAI) that was acquired from a corynephage, may also be responsible for the emergence of toxigenic strains causing recurrent infectious processes, since PAIs are very unstable genomic regions, which leads to fast acquisition/loss of genes (Tumapa et al., 2008). Besides, PAIs are acquired by horizontal gene transfer (HGT) events and harbor genes coding for virulence factors in pathogenic bacteria, and therefore, they are involved in the emergence of several bacterial pathogenic strains (Karaolis et al., 1998; Kauser et al., 2004; Gal-Mor and Finlay, 2006).

All together, these statements, along with the presence of 13 putative PAIs in C. diphtheriae NCTC 13129 and the appearance of nontoxigenic strains causing deaths, point to a great variability in the PAIs of this species. In fact, Iwaki et al. (2010) have demonstrated the deletion of 11 of the 13 PAIs in the non-toxigenic C. diphtheriae strain ATCC 27010, a standard strain used in several works. This high plasticity may reflect on bacterial life style and physiological versatility, and the results obtained through comparative genomic approaches can elucidate its importance to bacterial evolution (Dobrindt and Hacker, 2001).

The aim of the present study was to investigate the relationships between the great number of PAIs in C. diphtheriae and the possible implications of their plasticity on virulence.

The GenoFrag software was used to design primers to perform analysis based on the method plasticity of chromosome revealed by long range-polymerase chain reaction (PCR) (Ben et al., 2004, 2006). The software was pre-configured to design primers with 25 nucleotides and $60-65^{\circ} \mathrm{C}$ of melting temperature, keeping other parameters immutable. Primers that generated amplicons and overlapping regions of approximately 5.7 and $1.0 \mathrm{~kb}$, respectively, were chosen to perform the analyses since this length satisfactorily covers the extent of the third putative pathogenicity island of $C$. diphtheriae $(\mathrm{PiCd} 3)$.

PiCd 3 was chosen as a positive control since it is harbored by C. pseudotuberculosis 
(third putative pathogenicity island of C. pseudotuberculosis - PiCp 3), another pathogenic bacterium from the same genus, therefore making this PAI potentially important for virulence in the described genus. Besides, PiCd 3 and PiCd 5 seem to be more stable than the others, since they are the only PAIs harbored by C. diphtheriae ATCC 27010 (Iwaki et al., 2010). PiCd 8 was chosen as a model since it is substituted by another PAI in C. pseudotuberculosis (PiCp 5 ) with a different gene composition, which points to a putative "hotspot" for HGT. Furthermore, this island is one of the 3 PAIs that are concurrently absent on the $C$. diphtheriae strains ATCC 27010 and PW8 (Iwaki et al., 2010). Table 1 shows primers used to amplify regions inside the PiCds 3 and 8, designed from the genome sequence of C. diphtheriae NCTC 13129.

Table 1. Name, annealing position and nucleotide sequence of the primers used to amplify regions inside PiCds 3 and 8 .

\begin{tabular}{llllll}
\hline Name & Start & Nucleotide sequence & Name & End & Nucleotide sequence \\
\hline PiCd 3 1F & 250200 & 5'-AACGTCGACTTACCGGCCCCAGAAC-3' & PiCd 3 1R & 255934 & 5'-CTGGTTCTCACTGCGTGTGGGTCTG-3' \\
PiCd 8 1F & 1680348 & 5'-CCTCCGTCTCGATCACGACGAAGGT-3' & PiCd 8 1R & 1686105 & 5'-ACGGGAGACCGTCAACAGCTCGATG-3' \\
PiCd 8 2F & 1685049 & 5'-CCCCAGCATATTGGCCGTTGATGGG-3' & PiCd 8 2R & 1690786 & 5'-TCCAGTTGTCTCGCCGGGTAGAAGG-3' \\
PiCd 8 3F & 1690054 & 5'-AGCCTAGGCTTCCCCAGAACTCACG-3' & PiCd 8 3R & 1695784 & 5'-TCTGGTGGATGGGCTATTCCGGTGG-3' \\
PiCd 8 4F & 1694713 & 5'-AGACTCCGGTGTCCCTCAACACACC-3' & PiCd 8 4R & 1700440 & 5'-CTACCGCGATATGGCGAACACCTGG-3' \\
\hline
\end{tabular}

We used 11 C. diphtheriae strains: ATCC 27010 and ATCC 27012, standard strains for virulence studies of non-toxigenic and toxigenic strains, respectively; CDC E8392, from the Centers for Disease Control collection, and 8 Brazilian strains, 2 of them isolated from respiratory diphtheria (TR 241 and VA01) and 6 from invasive infections (814, 402 and HC01-04). ATCC 27010 was used as control.

C. diphtheriae strains were maintained in brain heart infusion (BHI) broth at $37^{\circ} \mathrm{C}$, with stirring. Afterwards, chromosomal DNA sequences were obtained using phenol-chloroform and 10\% lysozyme (Sambrook et al., 1989) and DNA concentrations were determined spectrophotometrically and on $0.8 \%$ agarose gels. Finally, amplifications were carried out in an MJ Research thermocycler as follow: 0.5-2 $\mu$ L DNA solution, $2 X$ Mix FideliTaq USB and 1 $\mu \mathrm{L} 100 \mu \mathrm{M}$ of each primer in a final volume of $25 \mu \mathrm{L}$ (sterile Milli-Q water). The mixture was denatured at $94^{\circ} \mathrm{C}$ for $2 \mathrm{~min}$, followed by 30 cycles of amplification at $94^{\circ} \mathrm{C}$ for $30 \mathrm{~s}, 60^{\circ} \mathrm{C}$ for $30 \mathrm{~s}$ and $72^{\circ} \mathrm{C}$ for $10 \mathrm{~min}$, with a final extension of $72^{\circ} \mathrm{C}$ for $12 \mathrm{~min}$.

Figure 1 shows amplification patterns using 1 set of primers for PiCd 3 and 4 sets of primers for PiCd 8. Nine of the 11 strains had PiCd 3 amplicons, corroborating the putative

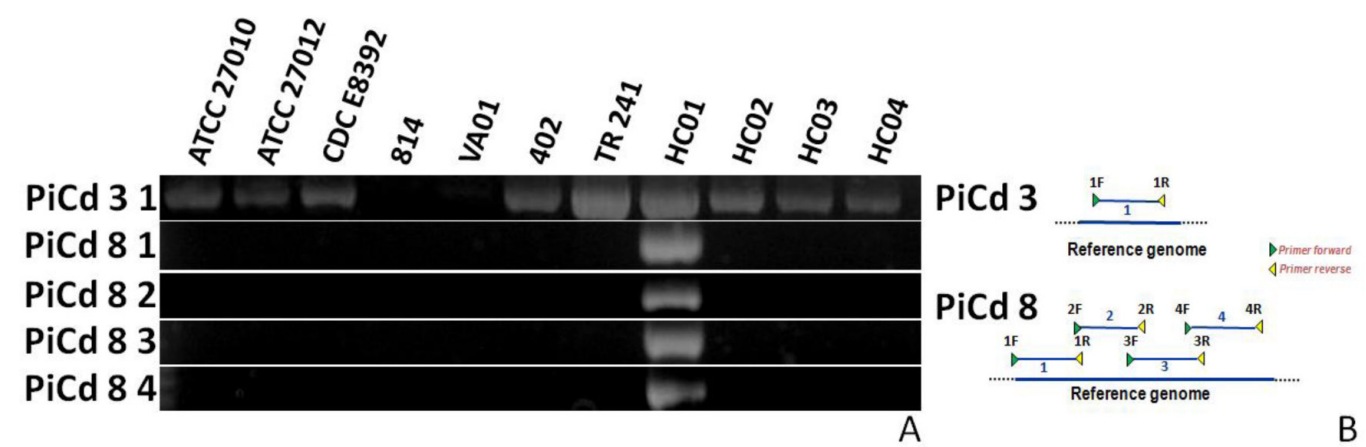

Figure 1. A. Electrophoresis gel exhibiting amplification patterns of PiCds 3 and 8. B. Graphic representation of primer annealing. PiCd 3 an PiCd 8 were amplified using 1 and 4 sets of primers, respectively. 
importance of this island for the species and, possibly, for the genus Corynebacterium. Only strains 814 and VA01 did not have PiCd 3.

PiCd 3 harbors genes that play important roles in virulence. The $a f u A B$ operon, a pit $A B$-like operon, is responsible for acquiring iron from proteins, aiming to overcome the restricted availability of iron within hosts. As main characteristics, the pitAB-like transporters target proteins such as transferrin, hemin and ferritin and are commonly harbored by PAIs, and, due to their importance, they are frequently present in several copies, so the deletion of one operon will not affect virulence drastically (Brown et al., 2002).

PiCd 3 also harbors the $g l p T$ and $t c S S 1-R 1$ genes. The $g l p T$ gene is responsible for mediating the transport of glycerol 3-phosphate across the membrane in bacteria and plays an important role during inorganic orthophosphate starvation (Enkavi and Tajkhorshid, 2010). Besides, the $g l p T$ gene is regulated by the $p h o B R$ genes, which show great similarity with the $t c s S 1-R 1$ genes. In addition, the pho regulon is directly involved in the regulation of virulence genes of several bacteria, including an Escherichia coli strain pathogenic to pigs (Ishige et al., 2003; Pratt et al., 2010; Yoshida et al., 2010). Finally, the importance of the genes present in PiCd 3 corroborates its greater stability.

Unlike PiCd 3, PiCd 8 was present in only one of the $C$. diphtheriae strains analyzed, the $C$. diphtheriae HC01. This pattern may be due to the total absence of the PAI or due to insertions of great amounts of DNA inside each of the four regions of PiCd 8 analyzed, which could prevent the amplification of the fragments. Anyway, the two hypotheses point to the instability of PiCd 8 and corroborate the assignment of this region as a putative "hotspot" for HGT events.

PiCd 8 presents a high concentration of hypothetical and putative genes, a common feature of PAIs (Hsiao et al., 2005). On the other hand, the island harbors a GntR-like transcriptional regulator that plays a pivotal role in bacterial growth under carbon starvation. The gnt $R$ product acts in the presence of gluconate to negatively regulate gluconate permeases and kinases that are responsible for transporting and phosphorylating gluconate, respectively, in order to use it as an additional carbon source (Frunzke et al., 2008). However, although gnt $R$ is important in bacterial growth, imposing an obstacle for its deletion, it is present as three copies along the $C$. diphtheriae genome.

Finally, the high stability of $\mathrm{PiCd} 3$ and the plasticity of $\mathrm{PiCd} 8$ may provide valuable information about the lifestyle of $C$. diphtheriae making the studies of other pathogenicity islands in this organism a very important approach to gather data for the development of more effective vaccines and treatments for the medical disease known as Diphtheria.

\section{ACKNOWLEDGMENTS}

Research supported by the Brazilian funding agencies CNPq (grant CNPq/MAPA), FAPEMIG and CAPES.

\section{REFERENCES}

Ben ZN, Gautier M, Andonov R, Lavenier D, et al. (2004). GenoFrag: software to design primers optimized for whole genome scanning by long-range PCR amplification. Nucleic Acids Res. 32: 17-24.

Ben ZN, Grimaldi C, Gautier M, Langella P, et al. (2006). Testing of a whole genome PCR scanning approach to identify genomic variability in four different species of lactic acid bacteria. Res. Microbiol. 157: 386-394.

Brown JS, Gilliland SM, Ruiz-Albert J and Holden DW (2002). Characterization of pit, a Streptococcus pneumoniae iron 
uptake ABC transporter. Infect. Immun. 70: 4389-4398.

Dobrindt U and Hacker J (2001). Whole genome plasticity in pathogenic bacteria. Curr. Opin. Microbiol. 4: 550-557.

Enkavi G and Tajkhorshid E (2010). Simulation of spontaneous substrate binding revealing the binding pathway and mechanism and initial conformational response of GlpT. Biochemistry 49: 1105-1114.

Frunzke J, Engels V, Hasenbein S, Gatgens C, et al. (2008). Co-ordinated regulation of gluconate catabolism and glucose uptake in Corynebacterium glutamicum by two functionally equivalent transcriptional regulators, GntR1 and GntR2. Mol. Microbiol. 67: 305-322.

Gal-Mor O and Finlay BB (2006). Pathogenicity islands: a molecular toolbox for bacterial virulence. Cell Microbiol. 8: 1707-1719.

Hsiao WW, Ung K, Aeschliman D, Bryan J, et al. (2005). Evidence of a large novel gene pool associated with prokaryotic genomic islands. PLoS Genet. 1: e62.

Ishige T, Krause M, Bott M, Wendisch VF, et al. (2003). The phosphate starvation stimulon of Corynebacterium glutamicum determined by DNA microarray analyses. J. Bacteriol. 185: 4519-4529.

Iwaki M, Komiya T, Yamamoto A, Ishiwa A, et al. (2010). Genome organization and pathogenicity of Corynebacterium diphtheriae C7(-) and PW8 strains. Infect. Immun. 78: 3791-3800.

Karaolis DK, Johnson JA, Bailey CC, Boedeker EC, et al. (1998). A Vibrio cholerae pathogenicity island associated with epidemic and pandemic strains. Proc. Natl. Acad. Sci. U. S. A. 95: 3134-3139.

Kauser F, Khan AA, Hussain MA, Carroll IM, et al. (2004). The cag pathogenicity island of Helicobacter pylori is disrupted in the majority of patient isolates from different human populations. J. Clin. Microbiol. 42: 5302-5308.

Mattos-Guaraldi AL, Moreira LO, Damasco PV and Hirata JR (2003). Diphtheria remains a threat to health in the developing world - an overview. Mem. Inst. Oswaldo Cruz 98: 987-993.

Nakao H, Pruckler JM, Mazurova IK, Narvskaia OV, et al. (1996). Heterogeneity of diphtheria toxin gene, tox, and its regulatory element, dtxR, in Corynebacterium diphtheriae strains causing epidemic diphtheria in Russia and Ukraine. J. Clin. Microbiol. 34: 1711-1716.

Popovic T, Mazurova IK, Efstratiou A, Vuopio-Varkila J, et al. (2000). Molecular epidemiology of diphtheria. J. Infect. Dis. 181 (Suppl 1): S168-S177.

Pratt JT, Ismail AM and Camilli A (2010). PhoB regulates both environmental and virulence gene expression in Vibrio cholerae. Mol. Microbiol. 77: 1595-1605.

Sambrook J, Fritsch EF and Maniatis T (1989). Molecular Cloning: A Laboratory Manual. 2nd edn. Cold Spring Harbor, Cold Spring Harbor Laboratory, New York.

Sharma NC, Banavaliker JN, Ranjan R and Kumar R (2007). Bacteriological \& epidemiological characteristics of diphtheria cases in \& around Delhi - a retrospective study. Indian J. Med. Res. 126: 545-552.

Tumapa S, Holden MT, Vesaratchavest M, Wuthiekanun V, et al. (2008). Burkholderia pseudomallei genome plasticity associated with genomic island variation. BMC Genom. 9: 190.

Yoshida Y, Sugiyama S, Oyamada T, Yokoyama K, et al. (2010). Identification and characterization of novel phosphate regulon genes, ecs0540-ecs0544, in Escherichia coli O157:H7. Mol. Genet. Genom. 284: 197-205. 